\title{
Semi-autonomous sounding selection for OCO-2
}

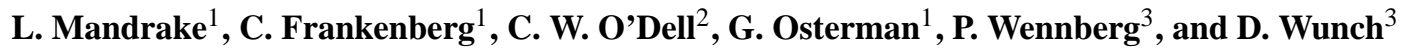 \\ ${ }^{1}$ Jet Propulsion Laboratory, California Institute of Technology, Pasadena, CA, USA \\ ${ }^{2}$ Colorado State University, Fort Collins, CO, USA \\ ${ }^{3}$ California Institute of Technology, Pasadena, CA, USA \\ Correspondence to: L. Mandrake (lukas.mandrake@jpl.nasa.gov)
}

Received: 10 June 2013 - Published in Atmos. Meas. Tech. Discuss.: 26 June 2013

Revised: 13 September 2013 - Accepted: 23 September 2013 - Published: 25 October 2013

\begin{abstract}
Many modern instruments generate more data than may be fully processed in a timely manner. For some atmospheric sounders, much of the raw data cannot be processed into meaningful observations due to suboptimal viewing conditions, such as the presence of clouds. Conventional solutions are quick, empirical-threshold filters hand-created by domain experts to weed out unlikely or unreasonable observations, coupled with randomized down sampling when the data volume is still too high. In this paper, we describe a method for the construction of a subsampling and ordering solution that maximizes the likelihood that a requested data subset will be usefully processed. The method can be used for any metadata-rich source and implicitly discerns informative vs. non-informative data features while still permitting user feedback into the final features selected for filter implementation. We demonstrate the method by creating a selector for the spectra of the Japanese GOSAT satellite designed to measure column averaged mixing ratios of greenhouse gases including carbon dioxide $\left(\mathrm{CO}_{2}\right)$. This is done within the Atmospheric $\mathrm{CO}_{2}$ Measurements from Space (ACOS) NASA project with the intention of eventual use during the early Orbiting Carbon Observatory-2 (OCO-2) mission. OCO-2 will have a 1.5 orders of magnitude larger data volume than ACOS, requiring intelligent pre-filtration.
\end{abstract}

\section{Introduction}

Atmospheric composition measurement, like so many modern disciplines, is undergoing a radical transformation due to new sensors. Ultra-high data rate, computerized instruments operate at hyper-spectral resolutions from space-borne platforms that operate over land and sea, day and night. Each data point was once a precious commodity carefully considered by multiple researchers; now an individual is swiftly presented with millions of observations and their associated metadata. A key challenge to handling the increased data volume is the estimation of data quality, to prevent the waste of precious human attention. Many remotely sensed atmospheric data suffer from unavoidable confounding aspects such as cloud cover or aerosols yielding distorted or unusable results given current retrieval algorithms. Certain observing conditions may a priori pose known problems for the processing algorithm, e.g. bright icy surfaces, dark oceans, or the complex surfaces of mountains (O'Dell et al., 2012). The instrumentation also contributes to difficulty in maintaining data quality, with drifting calibration, reset events, detector saturation or low-level preprocessor version changes making evaluation difficult. Poorly modeled physical processes also act as confounding forces in the observations where they dominate over the intended measurement theory during retrieval processing. When the data set is small, manual filtering of the data based on expert knowledge and past experience is a time-tested method for evaluation. But when millions of observations are faced, we must resort to an automated rules-based filtration without the advantage of personal interaction with each sounding.

The traditional method for determining such a filter proceeds by graphing a fully processed subset of the data versus various data metrics or features from the input metadata guided by expert experience. For instance, a spectral measurement may be categorized by metrics such as its overall intensity, the signal-to-noise ratio observed in a wellunderstood region, and known saturation indicators, as well as associated metadata such as the time, geometry, and various system parameters at the moment of measurement. The 
scientist then looks for thresholds in these candidate filtration features, beyond which the values change from reasonable to unreasonable based on prior knowledge, and visual features in the graph of feature versus desired output. A key question is how one knows the reasonable from the unreasonable, with the answer that only in rather egregious cases is the answer clear.

The resultant filter, often constructed from the amalgam of many "reasonability" thresholds, produces a cleansed data set that has flagged as questionable much of the obviously flawed data outliers. Some iteration is then performed by manually modifying the individual "reasonability" thresholds to alter the percentage of data passage to match the immediate needs of the project. This yields a final "fixed" filter with a given throughput and performance. The lack of ability to tune the filter in real time to increase or decrease throughput is one major shortcoming of such filters, as is the complexity of decoding why a particular data point has been excluded given several interacting potential filtration rules.

Generally, few scientific data sets are dominated by data that is entirely corrupt or acceptable. Instead, shades of acceptability for any particular data result from varying degrees of influence from confounding forces. Instead of constructing a binary good/bad filter, we observe the opportunity for a well-constructed data-screening algorithm: the ordering of the data by the statistical likelihood of each observation's scientific utility. Such an ordering could reproduce the above fixed filter performance by fixing the percentage of acceptance; beyond this, it permits the variation of the data passage rate in real time in a principled manner. In order to construct an estimate of a given observation's reliability, we turn to machine learning to emulate the same manual filter-construction process researchers traditionally perform on smaller data sets. By automatically constructing an ensemble of traditional, fixed filters, we may construct an estimate of the reliability of each data point based on the number of fixed filters that would reject it. It is important to recall we are describing a process that provides a robust, tunable filter, not merely a single static filter suggestion.

The satellite mission driving our development of new data filtering techniques is the Orbiting Carbon Observatory-2 (OCO-2) set to launch in 2014 with the intention of making atmospheric carbon dioxide $\left(\mathrm{CO}_{2}\right)$ measurements (Crisp et al., 2004; Connor et al., 2008; Boesch et al., 2011; O’Brien et al., 2011). OCO-2 will generate 24 soundings per second with each sounding comprised of $\sim 3000$ spectral radiance measurements, placing it soundly in the large data limit. The sophisticated physics-based retrieval algorithm (O'Dell et al., 2012) requires approximately $10 \mathrm{CPU}$ minutes $(3 \mathrm{GHz}$ Intel Xeon) to render each spectrum into a single retrieved Level 2 column $\mathrm{CO}_{2}$ value. This data volume will outpace real-time processing for anything less than $\sim 14000 \mathrm{CPU}$ cores, ignoring scaling inefficiencies that would increase the challenge. Even taking into account progress made to reduce the runtime of the OCO-2 Level 2 code as well as advances in hardware capability in advance of the expected 2014 launch date, selecting the "right" data to process will be critical. To satisfy the mission requirement that at least $6 \%$ of the soundings be processed in real time, it is likely that we must intelligently pre-select the soundings we attempt to process. This pre-selection of data with the highest scientific interest and minimal confounding influence is the definition of the sounding selection problem. We further wish to ensure we provide sampling criteria that maximize the utility of the OCO-2 data for estimation of carbon fluxes - the raison d'etre for the observatory. For instance, it is crucial that we sample $\mathrm{CO}_{2}$ at many atmospheric temperatures, pressures, seasons, and across many different Earth surface types, even if the current retrieval algorithm struggles in a region defined by such features.

\section{Data: GOSAT/ACOS example, pre-processing, features used}

In order to develop and test a method of sounding selector creation for the future OCO-2 mission, we use measurements of carbon dioxide taken by the Greenhouse gases Observing SATelite (GOSAT) provided by a joint effort between the Japanese Exploration Agency (JAXA), the Japanese National Institute for Environmental Studies (NIES) and the Japanese Ministry of the Environment (MOE). GOSAT measures in the same spectral regions as OCO-2 and provides a test bed for the OCO-2 retrieval algorithm (Yokota et al., 2004). The Atmospheric $\mathrm{CO}_{2}$ Observations from Space (ACOS) project creates a global carbon dioxide data product from the GOSAT observations. Processing the GOSAT soundings informed OCO-2 software development while the instrument was being built and integrated onto the spacecraft. We utilize ACOS/GOSAT soundings taken between 6 April 2009 and 30 September 2010, resulting in a set of 4.8 million soundings. We take only data recorded in the GOSAT "high gain" mode (Wunch et al., 2011; Crisp et al., 2012), as it is the dominant data type in number $(86 \%)$ and global coverage.

While the OCO-2 Level 2 retrieval software is used on the GOSAT data to generate the ACOS data set, the GOSAT L1 metadata is utilized directly, consisting of geospatial information and estimates of signal-to-noise provided by JAXA. The $\mathrm{L} 1$ radiance spectra are not directly used for developing a sounding selection scheme, but are used in retrieval preprocessing routines that then feed into the sounding selector. The $\mathrm{L} 1$ data are used in a cloud detection scheme (Taylor et al., 2012) and a combination cloud filter and $\mathrm{CO}_{2}$ quick-look based on a fast non-scattering retrieval algorithm (Frankenberg et al., 2005). No data rejected by the cloud detection scheme (Taylor et al., 2012) is retained, forming a pre-screen to remove the most offending cloud opacities. These two rapid preprocessing algorithms generate a variety of useful estimates and diagnostics such as albedo, surface pressure, temperature, the ratio of $\mathrm{CO}_{2}$ estimates as determined by 


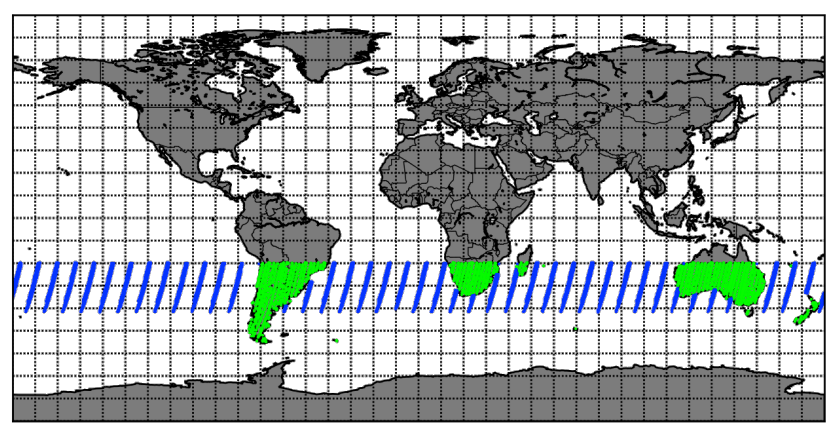

Fig. 1. The land and sea data used in the Southern Hemisphere approximation. This region is observed to have very low seasonal $\mathrm{CO}_{2}$ fluctuation relative to the north.

independent regions of the radiance spectrum. Finally, some statistical measurements of the radiance spectra are also calculated such as standard deviation, max, min, and mean. Together, these form the metadata and metric inputs (features) that we will use to divine a sounding's likelihood of being successfully retrieved. Table 1 shows the quantity, type and origin of the features used.

To guide our filter optimization, we seek to minimize the scatter in the final retrieved $\mathrm{CO}_{2}$ value produced by the full physics retrieval algorithm (here version B2.10), after the official cloud filtration algorithm has been applied (see Sect. 3.2.2). We focus on the low variation of atmospheric $\mathrm{CO}_{2}$ in the Southern Hemisphere $\left(20^{\circ}\right.$ to $60^{\circ} \mathrm{S}$, see Fig. 1). Applying these criteria results in 40000 retrievals over land and 24000 over ocean for training purposes.

\section{Method: hyper-dimensional filter, goal function, and genetic algorithm}

In this section we provide an overview of the methods to be used in the general case. We start by defining a large set of filters similar to those produced by experts. Each of these filters may use information from sounding metadata (a priori surface pressure and temperature, etc.) or direct spectral measurements (e.g. SNR, band ratios, etc.). We then utilize a genetic algorithm to optimize the thresholds of these filters, seeking to minimize the scatter in the retrieved $\mathrm{CO}_{2}$ in the Southern Hemisphere while maximizing the amount of data passed and minimizing the number of rules required in each filter. We produce 20 "warn levels" from 19 representative filters that summarize each sounding's acceptability to the larger set of filters, where a high warn level predicts most filters would reject a given sounding. The warn levels, in addition to spatial coverage requirements, form the basis of the sounding selector itself. The specific implementation of these methods is provided in Sect. 4 for the GOSAT B2.10 data set.

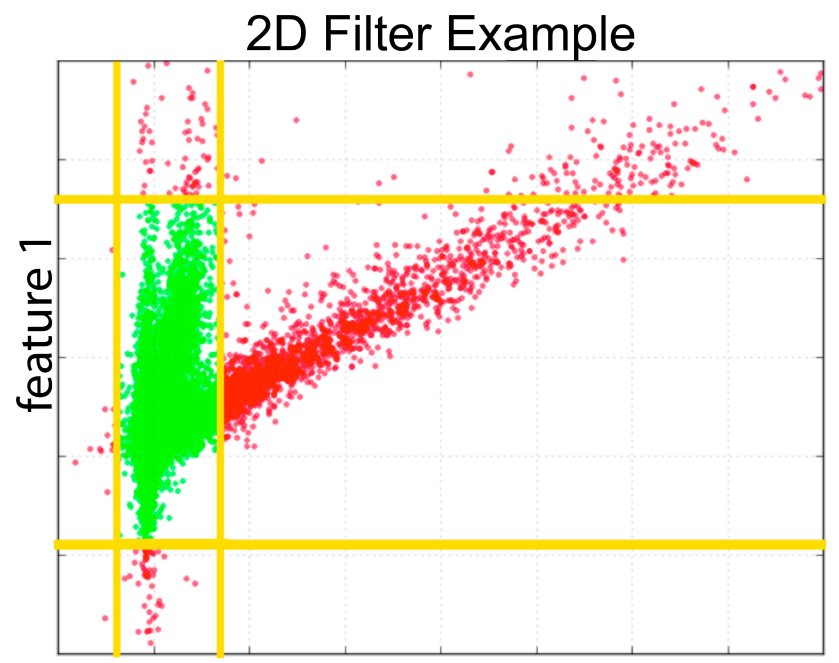

feature 2

Fig. 2. Two-dimensional feature example. Yellow lines represent the max and min thresholds for each of the two subfilters, with only data satisfying the union (green) accepted by the filter and all other data (red) rejected.

\subsection{Hyper-dimensional filter}

Following the "human expert" model of filter construction, we define a fixed filter as the union of a large number of simple threshold cutoffs (subfilters) each defined by a maximum and minimum threshold value beyond which data will be rejected (Fig. 2). One such subfilter is required for each input feature. Optimizing the high and low threshold for each subfilter defines the fixed filter creation problem, and the entire list of thresholds (number of input features $\times 2$ ) uniquely defines a fixed filter and is a high dimensional parameter space. A human expert generally creates one or two fixed filters, with the goal of segregating high, medium, and poor data quality or simply pass and fail. The full sounding selector will be based on an ensemble of 19 fixed filters, each with their own optimized subfilters. A guiding diagram is provided in Fig. 3.

\subsection{Genetic algorithm}

Genetic algorithms (GA) are methods that mimic natural selection to explore high dimensional parameter spaces (Periaux and Galan, 1995). They do not guarantee a globally optimal solution, but given the imprecise definition of perfection in many real-world optimization problems, such local minima often suffice or can be easily escaped with random restarts and well-chosen mutation. GA's are also quite simple to create and use, and they lend themselves trivially to highly parallel processing such as multicore and clustered computer systems. We will use a GA to optimize the high dimensional parameter space of the subfilter thresholds that make up each of our fixed filters. A guiding diagram is provided in Fig. 4. 
Table 1. Features considered as input to the GOSAT sounding selector construction.

\begin{tabular}{rlll}
\hline Quantity & Type & Example & Source \\
\hline 8 & Physical & $T_{\text {surf }}, P_{\text {surf }}$ & Cloud Filter Pre-process \\
5 & Signal Characteristics & SNR & Cloud Filter Pre-process \\
5 & Physical & CO $_{2}$ density & IMAP-DOAS Pre-process \\
12 & Spectral Stats & Stdev(radiance) & Spectral math on radiance spectra \\
30 & Signal Characteristics & SNR & JAXA \\
43 & Geometry & Azim, Alt, fraction land $_{\text {land }}$ & JAXA \\
103 & & & Total \\
\hline
\end{tabular}

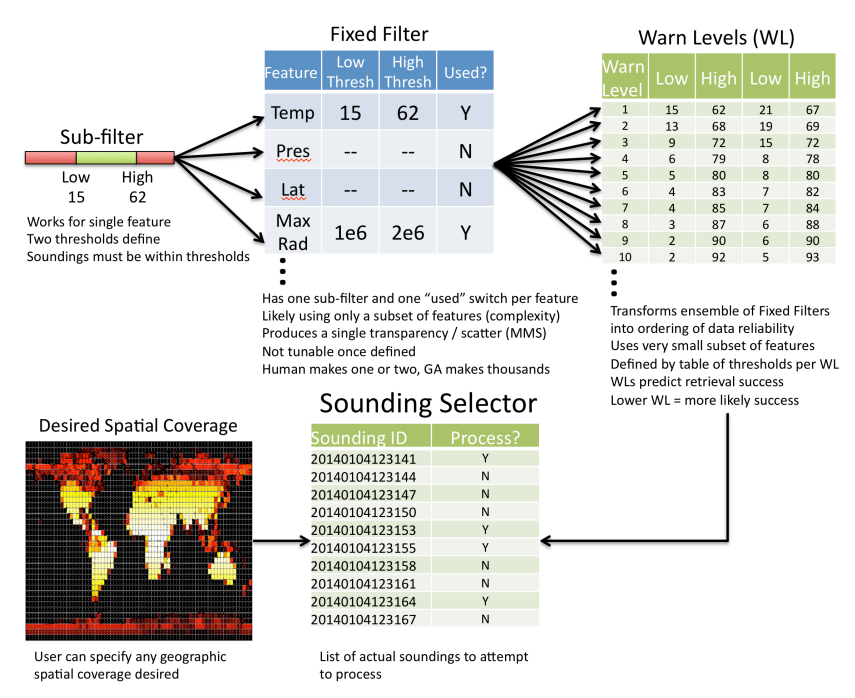

Fig. 3. A guiding diagram for the discussion of filters and the final sounding selector. Note that the double "Low, High" thresholds in the upper right table show that each of the Warn Levels is crafted from the combination of more than one Fixed Filter (in this example, two). Thus, two low and two high thresholds will be required given two input features. The color scale in the Desired Spatial Coverage to the lower left indicates the user-specified sounding density in each bin.

\subsubsection{Gene}

The first step of defining a GA is formulating the parameters (gene) that encapsulate the problem to be solved. In our case, we use the full list of subfilter high and low thresholds defined in Sect. 3.1 along with a Boolean list of whether each subfilter is actively being used. This list will permit us to track and control how many subfilters are actually used in each fixed filter, with the total number of active subfilters called a fixed filter's complexity. Initially, we start with a filter that permits all data and uses no active subfilters, thus complexity $=0$. A gene that only had two subfilters switched on would be complexity 2 , and all subfilters active would result in a complexity equal to the total number of features. Fixed filters constructed by humans are often highly complex by this definition, as they can have dozens of active subfilters.

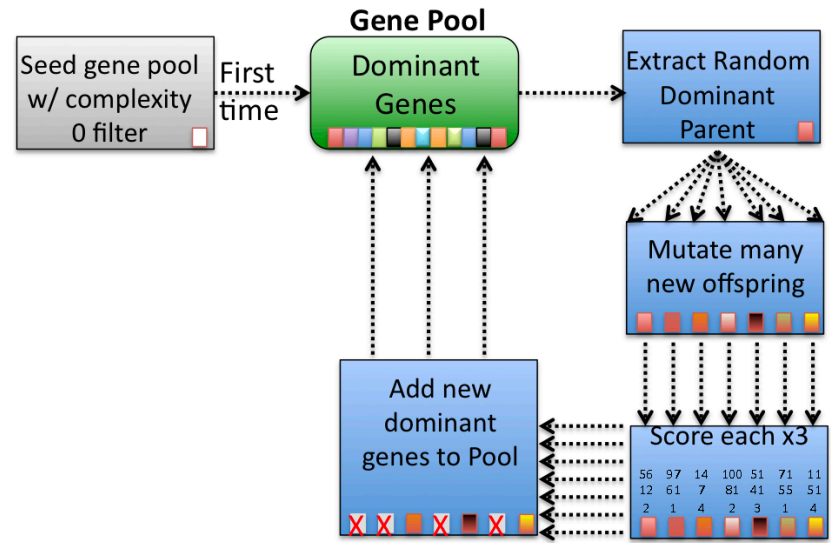

Fig. 4. The genetic algorithm (GA) cycle. A parent is selected from the dominant gene pool, mutated into thousands of offspring, and any new dominant solutions are added back into the pool while the others are discarded. This cycle can be run massively in parallel, with each process contributing to the same ever-improving gene pool.

\subsubsection{Fitness \& goal function}

Next we define the fitness by which each proposed genetic solution is judged. This, coupled with the definition of the gene in Sect. 3.2.1, fully defines the problem. Optimally, we would attempt to minimize the RMS difference between our retrieved $\mathrm{L} 2 \mathrm{CO}_{2}$ values and the true $\mathrm{CO}_{2}$ value over the entire globe across many years. Global $\mathrm{CO}_{2}$ observations from satellites exist but are not ideal for comparison to ACOS data and high quality surface data are spatially sparse (Wunch et al., 2011; Reuter et al., 2011). Instead, we utilize the Southern Hemisphere approximation: the annual $\mathrm{CO}_{2}$ variation of the Southern Hemisphere appears to be relatively small and reasonably approximated by a secular trend (linear yearly increase) with a very small sinusoidal seasonal component (Wunch et al., 2011). Most of the scatter seen in the Southern Hemisphere ACOS data is most likely caused by confounding forces negatively affecting the retrieval algorithm rather than actual $\mathrm{CO}_{2}$ fluctuation (Wunch et al., 2011). This has been shown to strongly support TCCON comparison on a global scale, and indeed our own experiments have shown 


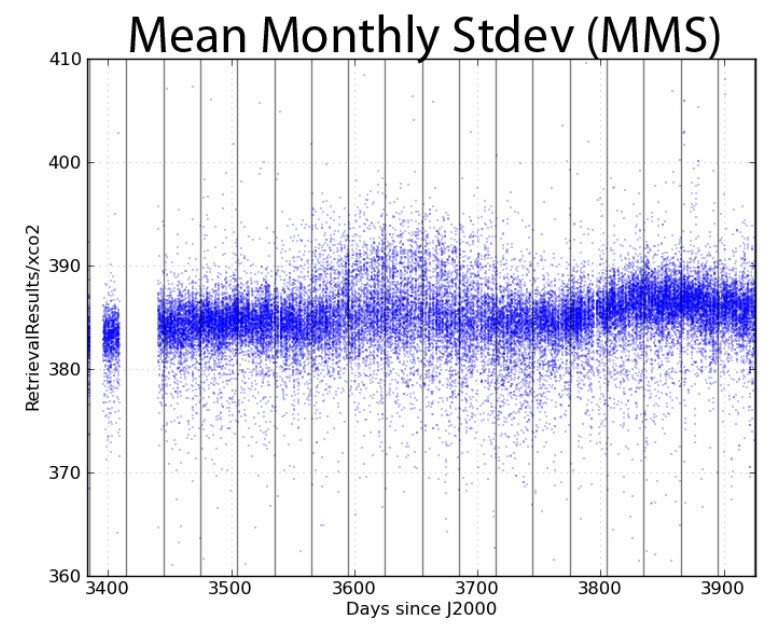

Fig. 5. A heat map of the retrieved values of $\mathrm{CO}_{2}$ for the entire Southern Hemisphere over land is shown. The blue bars identify the monthly bins within which the standard deviation will be taken. The MMS is the mean of each of the standard deviations of these 18 bins.

that creating a filter using TCCON comparison or Southern Hemisphere approximation yields extremely similar results.

We create a metric called mean monthly standard deviation (MMS):

MMS $=\frac{\sum_{\text {monthly_bins }} \operatorname{Stdev}\left(\mathrm{CO}_{2}\right)}{\text { num_monthly_bins }}$,

where only monthly bins with more than ten soundings are admitted into the above average (see Fig. 5 for a graphical example). Measured in parts per million (PPM), the same unit as $\mathrm{CO}_{2}$, it represents an overall scatter for the entire set of $x \mathrm{CO}_{2}$ measurement from ACOS in the Southern Hemisphere. The monthly grouping is to avoid any minor seasonal oscillations present in the data as well as the increase in $\mathrm{CO}_{2}$. Reducing the MMS through data filtration will be considered our primary goal.

The trivial solution to reduce MMS would be to filter all but a few very consistent soundings in a single monthly bin. In order to make this trade-off explicit, we will label fixed filter solutions with the percentage of data passed through the filter (transparency). Measured to tenths of a percent, this becomes a discrete quantity fundamental to the construction of our ensemble. Another goal will be to reduce the number of active subfilters necessary to achieve a solution or the complexity (also a discrete quantity). This allows for the prevention of overly complex fixed filters requiring dozens of subfilters when a very small number might perform almost as well. GA's handle multiple goals like these gracefully through the concept of dominance, as discussed in the next section.

\subsubsection{Dominance}

When evaluating a new, proposed fixed filter's fitness, we compute its MMS, transparency, and complexity. Transparency is resolved to $0.1 \%$ resolution, resulting in 1000 bins for the full range of 0 to $100 \%$. We then compare its MMS to a small population of past most-fit solutions called the Gene Pool. Within the Gene Pool past solutions are grouped by the discrete labels of transparency and complexity, ranked by resulting MMS. Only if the new proposed fixed filter has a lower MMS for a given complexity and transparency does it dominate (replace) the prior solution and continue into the next generation. In this way, we are running thousands of simultaneous races, 1000 transparency bins multiplied by the maximum complexity we will tolerate, that being 10 in the GOSAT example.

The net result of this process is not to find a single best fixed filter as a human expert might seek, but to form a population of dominant solutions that cannot be further reduced without specifying preferences between the three fitness metrics of transparency, complexity, and MMS. This population is known as the Pareto optimal front in machine learning circles (Jin and Sendhoff, 2008). Instead of seeking to arbitrarily judge between these three conflicting goals, we will preserve the trade-off space of equally fit fixed filters to help satisfy other requirements of our overall filter solution such as spatial coverage and tunability.

\subsubsection{Reproduction}

Reproduction in the GA describes the creation of new candidate filters, as shown in Fig. 4. Drawing randomly from the current gene pool of dominant fixed filters, each with their own MMS, transparency, and complexity, we propagate the chosen parent thousands of times. Each propagation is mutated; that is, random, unique changes are made to its subfilter thresholds and list of active subfilters. The fitness of the new candidate fixed filter is then measured with our three metrics. Most mutations will result in a less than dominant solution, and those candidates are discarded. A few will result in new, dominant candidates that will further fill the pool of dominant filters and/or replace candidates already within.

Different types of mutations are employed, also randomly. For instance, the mutation may change many subfilter thresholds at once or just a single entry. The threshold change may be a minor incremental alteration up or down, a large change up or down, or a totally random overwrite of the previous value. Combining many scales of mutations among the new candidate population permits us to escape local minima through occasional radical mutations while also permitting fine-tuning of nearly optimal filters through small mutations. We did not employ crossover operators (Periaux and Galan, 1995) as simpler mutations were found sufficient. After hundreds of iterations and millions of candidate fixed filters considered, the gene pool of dominant solutions becomes 
stable, that is minimally changing from cycle to cycle, and we achieve Pareto optimality.

\subsubsection{Termination}

The GA is permitted to run until the known dominant filters in the gene pool do not substantially change, i.e. the MMS for any particular transparency and complexity reduces by less than $0.01 \%$ per hundred GA cycles. In practice, this was a very stringent condition and looser definitions of termination would have resulted in similarly acceptable filter solutions.

\subsection{Feature selection}

Feature selection is the process of determining which of the input features are useful or informative for a given problem (Guyon and Elisseeff, 2003). In our example, we seek to answer which of the initial features aid us in minimizing the Southern Hemisphere scatter. The GA has produced several thousand dominant fixed filters that trade off MMS, transparency, and complexity. By examining the subset of the initial features selected by the GA in these dominant filters, we create a list of known informative features. Those not selected are either redundant, containing the same information as the chosen set, or are uninformative.

As we did not a priori restrict the choice of data features, each fixed filter might (but likely does not) employ totally different features in its functioning even for small changes in performance metric. This is exacerbated by the presence of highly correlated or equivalent features in our input data, such as the altitude of a sounding using two differing Earth spheroid estimates or multiple estimates of surface pressure using differing assumptions. Though the GA considers such nearly equivalent features as interchangeable and will flutter between them from fixed filter to fixed filter, some are often more interpretable than others. For example, two features commonly interchanged are the SNR and the standard deviation of the radiance spectrum. The SNR is straightforward to interpret, whereas the standard deviation of intensity convolves the SNR, overall brightness, and other more subtle quantities. Therefore, the set of informative features resulting from the GA is a superset that likely contains highly correlated, interchangeable features. We may take advantage of this apparent nuisance, hand-selecting out features from the GA-generated list that are (A) highly informative, (B) appear many times in the dominant fixed filters, and $(C)$ are most interpretable. A second run of the GA would then be performed using only this fine-tuned list of desirable features, regenerating the full dominant population with improved interpretability and minimized interchangeable feature oscillation. As such, this method is semi-autonomous rather than fully automatic, as human intervention has been permitted to aid in the fine-tuning and sanity checking of the features used in the final, dominant fixed filter solutions. However, the human expert in this case has already been aided by the
GA's focus on informative features in a data-driven, empirical manner.

\subsection{Warn level}

We now need a way to utilize the knowledge contained in the ensemble of dominant fixed filters. The human expert standard method would pick a single fixed filter that had the MMS reduction and transparency desired, using personal judgment to perform the balance between these goals. We seek to make a more complex, tunable sounding selector that benefits from more of the information stored in the ensemble than a single fixed filter instance. One way to encode this information is to order the data set from first to last rejected by the ensemble of filters as we sweep from 100 to $0 \%$ transparency. Such an ordering is the very definition of a sounding selector. Unfortunately, it also requires running several thousand filters on each sounding to generate its rank.

To reduce this computation and complexity load, we can sample the ensemble of fixed filters uniformly in transparency, say at $5 \%$ resolution. This makes our ordering nonunique, instead forming groups of equally trusted data. Each group may be labeled with the total number of the sampled fixed filters that would block all members within the group. We call this label the warn level (WL) of the group and all the data points within. $\mathrm{WL}=0$ indicates data that is unconditionally accepted by all sampled fixed filters, while WL equal to the number of filter samples (19) indicates data that is least acceptable. Relatively high WL indicates the data will likely not process into a useful $\mathrm{CO}_{2}$ value.

The WL values are themselves useful for data investigation, as with them one could request all the data that is highly likely or unlikely to process and study the spatial or temporal distribution, bias with respect to known truths, etc. These distributions will certainly not all be uniform, given that confounding forces are often local. However, the WL's original utility is in assisting sounding selection.

\subsection{Spatial coverage}

A required trait of a successful sounding selector is guaranteed spatial coverage. It is generally required that the globe be populated with representative, reliable data, with the understanding that certain spatial regions may be more problematic (e.g. cloud contamination in the tropics limits the number of successful retrievals). A fixed filter solution will not itself perform this function; in fact, it is unlikely we wish this. It is useful information to learn that over the stormy, shiny ice of Antarctica we always perform poorly relative to the rest of the planet. At the same time, we do not wish to require a global filter transparency of $80 \%$ or higher before we begin to admit Antarctic data.

The OCO-2 project requires that the spatial coverage system must operate on a per-granule (continuous fragment of an orbit) basis for ease of implementation. We segregate 


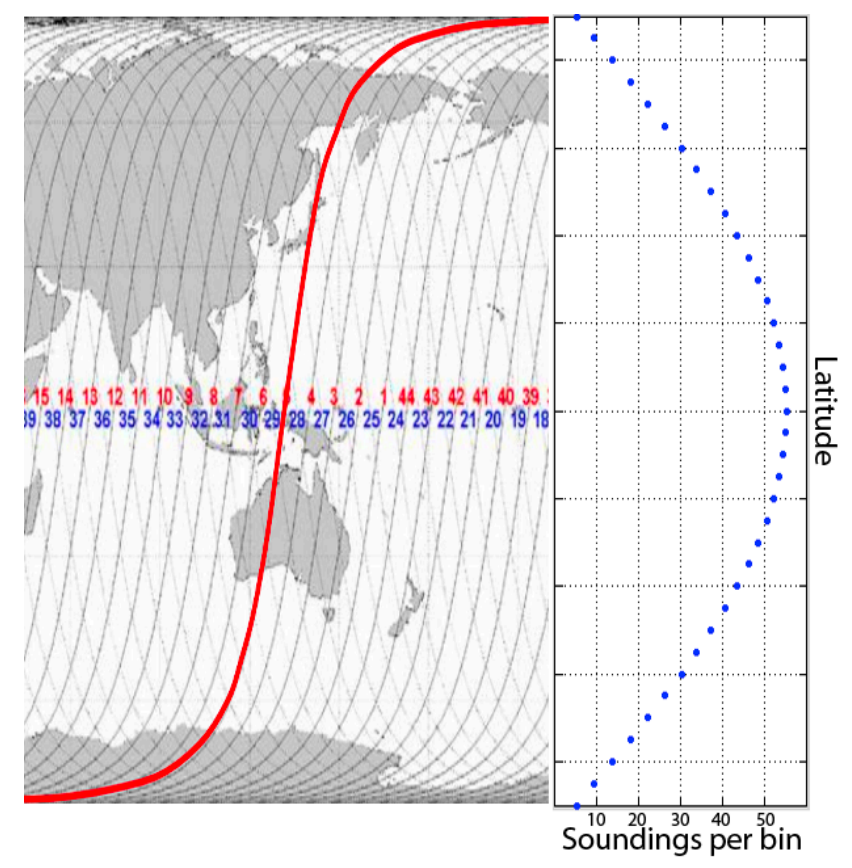

Fig. 6. On the left, a sample GOSAT track, used with permission from the GOSAT path calendar. The sounding selector would be applied to this track individually, with a user-specified fraction of the soundings selected for processing. On the right, an example distribution for the user-specified number of soundings per latitude bin desired.

these orbital tracks into latitudinal bins of 5 degrees. Within each of these bins, we may specify how many soundings we wish to accept (see Fig. 6). Generally, the number of requested soundings per bin is proportional to the cosine of latitude to prevent an overabundance of soundings near the poles. The spatial coverage system fills each latitude bin starting with the most trusted data as judged by its WL, accepting less trusted soundings as needed until the bin is filled. The list of soundings used to fill the bins is the output of the sounding selector.

\section{Results}

\subsection{Trade off curves (Land \& sea)}

Employing the above-detailed GA on the GOSAT data in the Southern Hemisphere resulted in a trade-off curve set for each of land (nadir mode) and sea (glint mode) data sets as shown in Figs. 7 and 8. These are the dominant gene pool solutions after extensive GA cycling for the two data sets. Approximately $1000 \mathrm{CPU}$-hours were spent on each data set using $3 \mathrm{GHz}$ Intel Xeon processors. Each point on these curves is an individual, dominant fixed filter. Each is identified by its transparency ( $x$ axis), complexity (color), and the resulting MMS of passed data ( $y$ axis). The land is seen to have

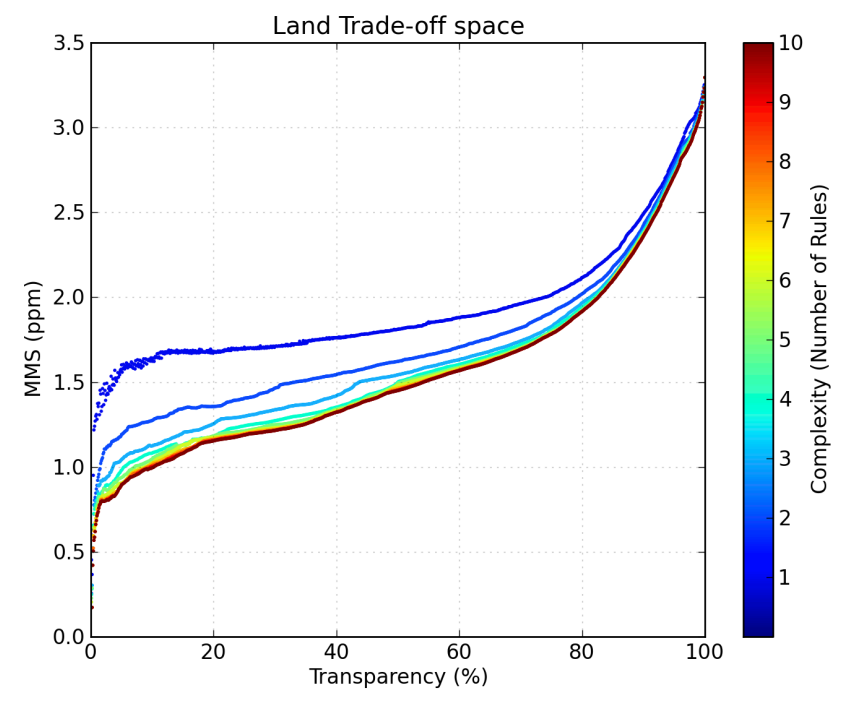

Fig. 7. The Pareto-optimal trade-off curves for dominant Land fixed filters in the Southern Hemisphere using all available features. Note that beyond a complexity of four, there is little filtration improvement. This means that four input features capture all of the filtration/prediction capability of all provided metadata, and all other features are either correlated with these four or are not predictive.

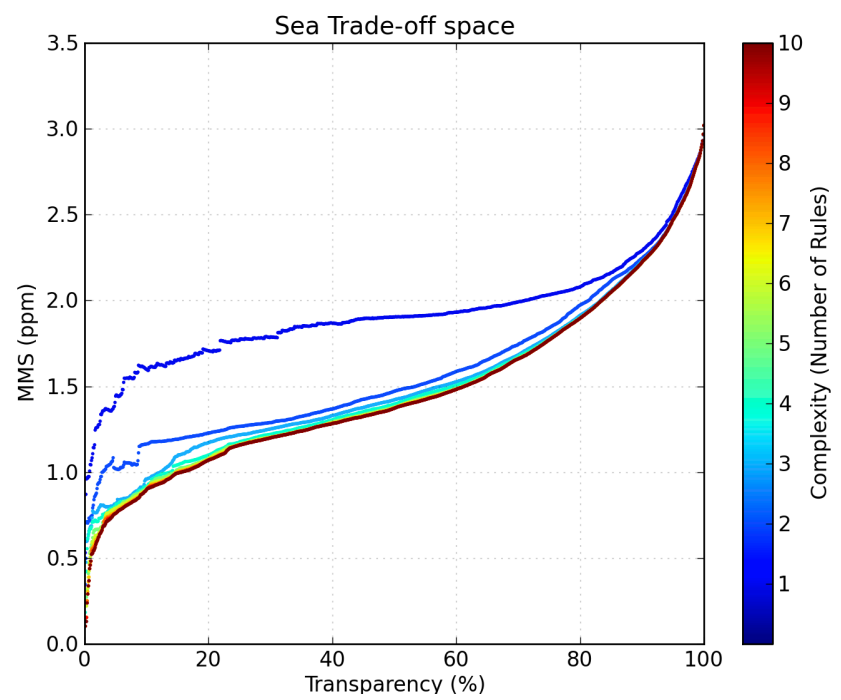

Fig. 8. The Pareto optimal trade-off curves for dominant Sea fixed filters in the Southern Hemisphere using all available features. Note that beyond a complexity of three, there is little filtration improvement.

a higher scatter than sea at every transparency, owing to its more varied and complex surface.

The slope of the trade-off curve is of vital interest to filter efficiency. Regions of zero MMS vs. transparency slope would show no effective reduction in southern scatter as we reduce the amount of data permitted through the fixed filter, or in other words poor filter performance. Filters with complexity equal to one are seen to possess such regions, whereas 
Table 2. Features chosen by the genetic algorithm to reduce scatter over Land (Nadir mode).

\begin{tabular}{cccll}
\hline Utility & Interpret & Restrict & Name & Source \\
\hline $59 \%$ & Med & Low & $\mathrm{CO}_{2}$ ratio & IMAP-DOAS Pre-process \\
$53 \%$ & Med & Low & Delta Pressure Cloud & Cloud-filter Pre-process \\
$40 \%$ & High & Med & Solar Zenith & JAXA Geometry \\
$9 \%$ & Med & Low & $\mathrm{H}_{2} \mathrm{O}$ ratio & Frankenberg Pre-process \\
$9 \%$ & High & Med & STDEV(Sounding Altitude) & JAXA Geometry \\
\hline
\end{tabular}

Utility measures the \% contribution of a given feature to the overall filter performance. Interpret qualitatively measures how easily interpretable a researcher finds the feature. Restrict captures how restrictive to the resulting database a subfilter based on this feature would be.

Table 3. Features chosen by the genetic algorithm to reduce scatter over Sea (Glint mode).

\begin{tabular}{cccll}
\hline Utility & Interpret & Restrict & Name & Source \\
\hline $67 \%$ & Med & Low & Delta Pressure Cloud & Cloud-filter Pre-process \\
$39 \%$ & Med & Low & $\mathrm{CO}_{2}$ ratio & IMAP-DOAS Pre-process \\
$27 \%$ & High & Med & Sounding cross-track angle & JAXA Geometry \\
$13 \%$ & High & Low & Min(weak $\mathrm{CO}_{2}$ radiance S-polar) & Spectral math \\
\hline
\end{tabular}

higher complexity filters do not. MMS instability at the lowest transparencies is normal, as so few soundings are permitted through the filter that low-N statistics begin to dominate the scatter calculation. High transparency filters are seen to be extremely efficient by their slope, as it is an easier problem to eliminate the most egregious outliers initially.

Higher complexity is seen to reduce scatter at a fixed transparency due to its ability to more precisely fit the data with more parameters and access additional feature information; however, using complexities greater than four for land and three for sea are seen to have strongly diminishing returns. As we desire to maintain a high degree of interpretability of the sounding selector's function, we prefer lower complexity. We choose to limit our complexity to two at this stage for the GOSAT example, for ease of graphing our results and interpretability in this example as well as satisfactory filter performance. However, one could in principle use a complexity four filter with only minor additional effort. The future OCO-2 mission will likely employ a two-tofour complexity filter, as prescribed by its empirical tradeoff curves. Note that at this stage, a complexity two filter might utilize many input features, but no more than two at any particular transparency. As an example, $60 \%$ of the filter's transparency might be comprised of the pairing of signal to noise ratio and co2_ratio, while the remainder of the curve might be constructed by the joining of $\mathrm{co}_{2}$ ratio and h2o_ratio. Three features would then be used, but only two at a time, thus complexity two. In the next section, we will impose the constraint of matching the total number of input features to the filter complexity to remove this detail.

\subsection{Feature selection}

\subsubsection{Interpretability vs. utility}

The fixed filters populating Figs. 7 and 8 were constructed from the entire set of available features provided to the GA. Features that were selected as most informative in the tradeoff curves are shown in Tables 2 and 3. Several new metrics are now defined to aid in the human semi-autonomous feature selection task. Utility is an estimation of the discriminating power of a feature to reduce scatter. To calculate it, we examined the subfilter thresholds for all fixed filters of complexity four. The percentage of all filtered soundings for which a feature is responsible is its utility. This is an overestimate of a feature's true usefulness, as other features may also be capable of filtering a particular point even if this feature were not present. For this reason, the utilities will sum to greater than $100 \%$ due to feature filter overlap. Only features with utility greater than $10 \%$ are reported to avoid large numbers of rarely useful features. Interpretability is a qualitative measure of the physical meaning of a particular feature. Deep, internal parameters from a preprocessor can have very opaque real-world connections resulting in a low interpretability, whereas physically measurable quantities are immediately understandable with high interpretability. Interpretability is vital for two reasons: it facilitates the future improvement of the retrieval algorithm by communicating to researchers some areas of potential difficulty, and it helps explain why some soundings are more challenging than others to future data users. Restriction is the qualitative tendency for a feature to eliminate soundings that are likely to be interesting to future analysis, or a feature that is commonly plotted against. Simple geometric/geographic cut-offs 

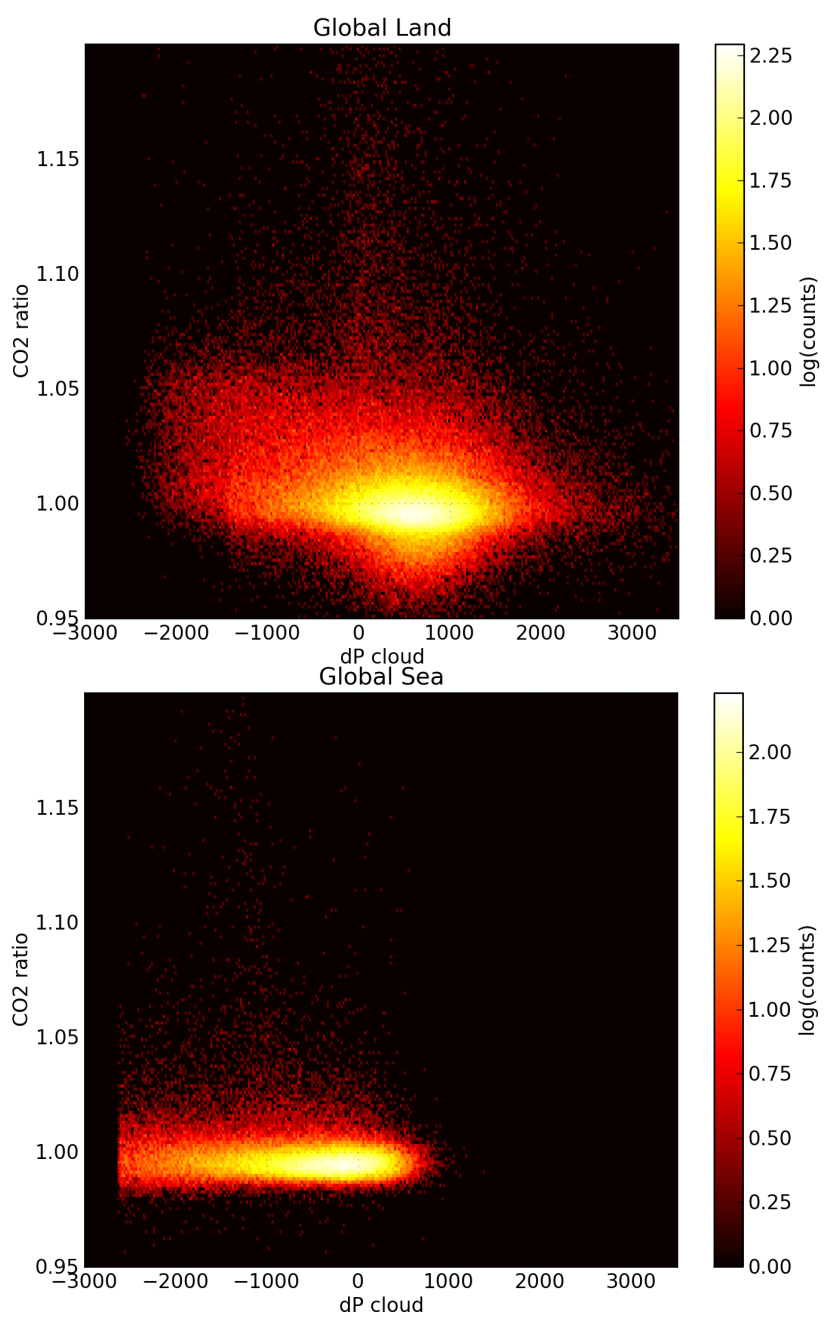

Fig. 9. Land/sea sounding distributions for Delta Pressure Cloud (dPc) versus $\mathrm{CO}_{2}$ ratio.

are less desirable for this reason, as researchers often wish to observe the dependence of a measurable as a function of various angles or altitudes. Also problematic are features that are highly correlated with $x \mathrm{CO}_{2}$, as these might preclude regions with true, large fluxes both positive and negative.

Comparing Tables 2 and 3 shows two features as highly significant (high utility, acceptable interpretability, and low restriction) in both land and sea cases: the $\mathrm{CO}_{2}$ ratio (CO2r, unitless) and Delta Cloud Pressure (dPc, in $\mathrm{hPa}$ units). The first feature derives from the Iterative Maximum A Posteriori Differential Optical Absorption Spectroscopy (IMAP-DOAS) preprocessor in which $\mathrm{CO}_{2}$ concentration estimates are independently derived for the strong and weak $\mathrm{CO}_{2}$ bands within the radiance spectrum using a non-scattering algorithm (Frankenberg et al., 2005). These estimates are not as robust as the GOSAT full physics retrieval algorithm but orders of magnitude faster to compute. The unitless ratio of $\mathrm{CO}_{2}$ estimates from both the strong

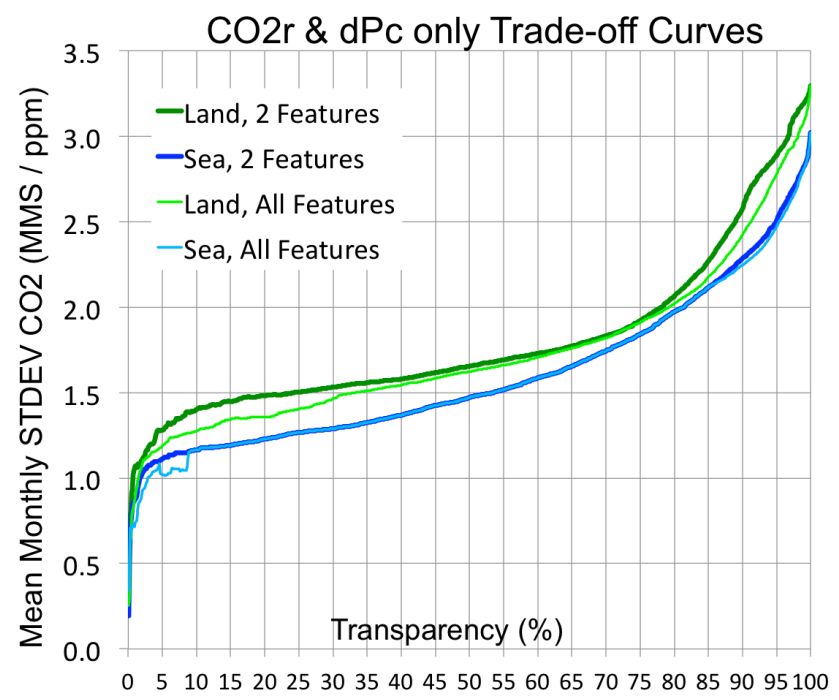

Fig. 10. Using only the two chosen features $\mathrm{CO}_{2}$ ratio $(\mathrm{CO} 2 \mathrm{r})$ and Delta Pressure Cloud $(\mathrm{dPc})$, the complexity $=2$ filters for land and sea are re-derived. Performance of the new 2-feature trade-off curves is similar to those using all-features, proving our feature selection was successful. The small dip observed in the "Sea, All Features" curve near $5 \%$ transparency is merely a subregion where the cross-track sounding angle paired with $\mathrm{CO} 2 \mathrm{r}$ was slightly more efficient than dPc plus $\mathrm{CO} 2 \mathrm{r}$.

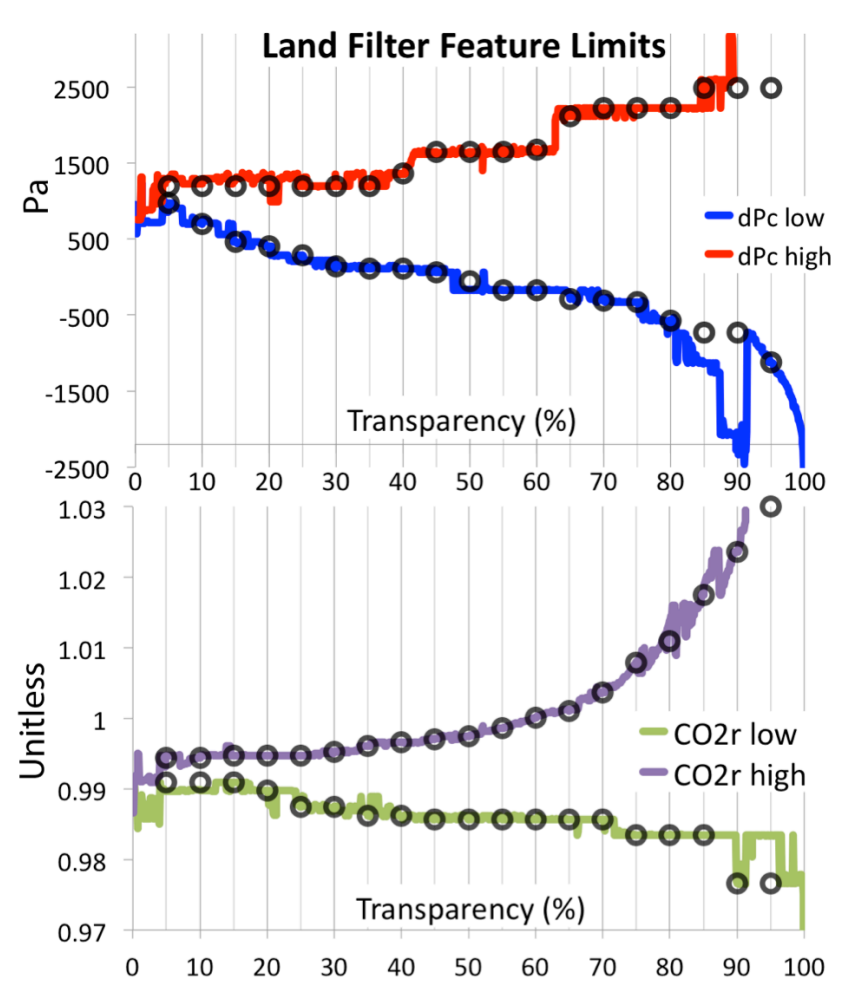

Fig. 11. Land data subfilter thresholds for $\mathrm{dPc}$ (top) and $\mathrm{CO} 2 \mathrm{r}$ (bottom). Black circles show monotonic, hand-smoothed final subfilter values. 


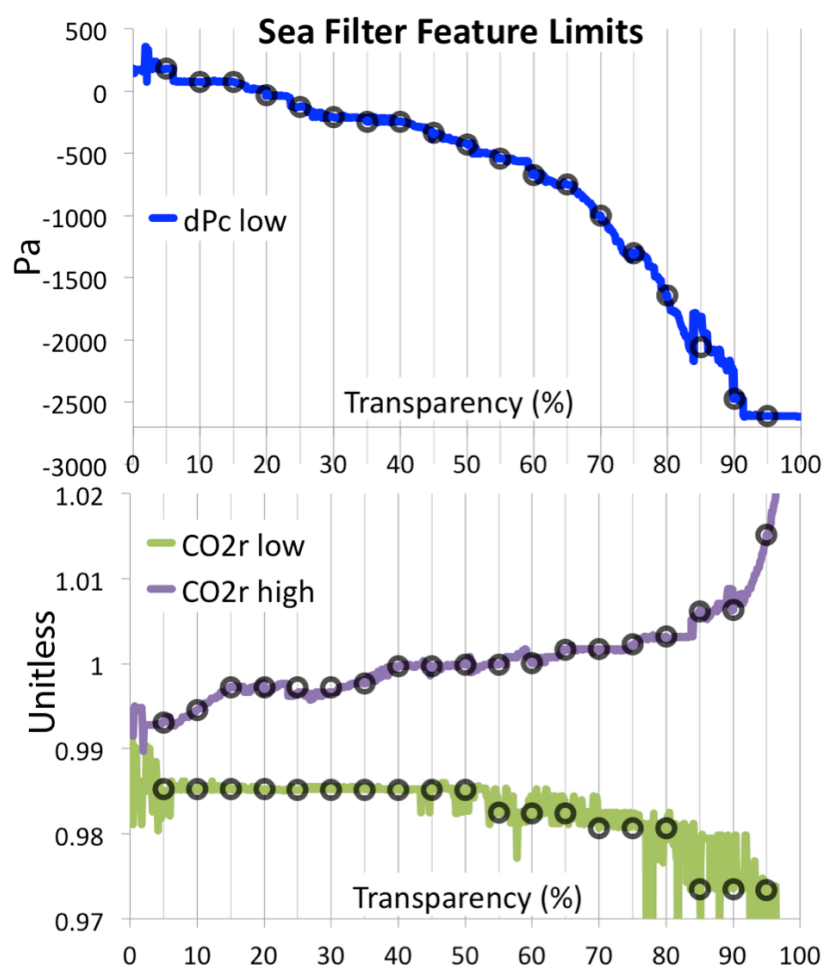

Fig. 12. Sea data subfilter thresholds for dPc (top) and CO2r (bottom). Black circles show monotonic, hand-smoothed final subfilter values. Note that the high threshold for $\mathrm{dPc}$ was not needed, as can be explained in Fig. 7, bottom.

and weak $\mathrm{CO}_{2}$ bands, $\mathrm{CO} 2 \mathrm{r}$, indicates enhanced scattering within the atmosphere when different from 1.0. It is intuitively satisfying that a spectral quality measure was selected as most useful. The second significant feature derives from the cloud filter preprocessor (Taylor et al., 2012), using the Oxygen A-band to derive surface pressure and goodness of fit assuming a Rayleigh-only atmosphere. The $\mathrm{dPc}$ is the difference between the preprocessor's quick-look surface pressure retrieved and the a priori surface pressure as given by the European Centre for Medium-Range Weather Forecasts (ECMWF) model. This parameter is critical in the determination of whether a sounding is too cloudy to process, as reflective cloud tops will retrieve a substantially lower pressure than anticipated. Not only is this reasonable as a sounding selector input, but it speaks to the diffuse boundary between clear and cloudy sounding determination.

Other features we will not use that still yield insight are the geometric factors of solar zenith angle (shallow illumination), cross-track angle (higher air mass), and standard deviation of the altitude within the sounding (mountainous or highly sloped regions with ill-defined ray paths). All of these are highly interpretable and reasonable inputs for a sounding selector, lending evidence that the GA did indeed select appropriate features and that our Southern Hemisphere approximation/scatter reduction proxy for the truth is functioning.
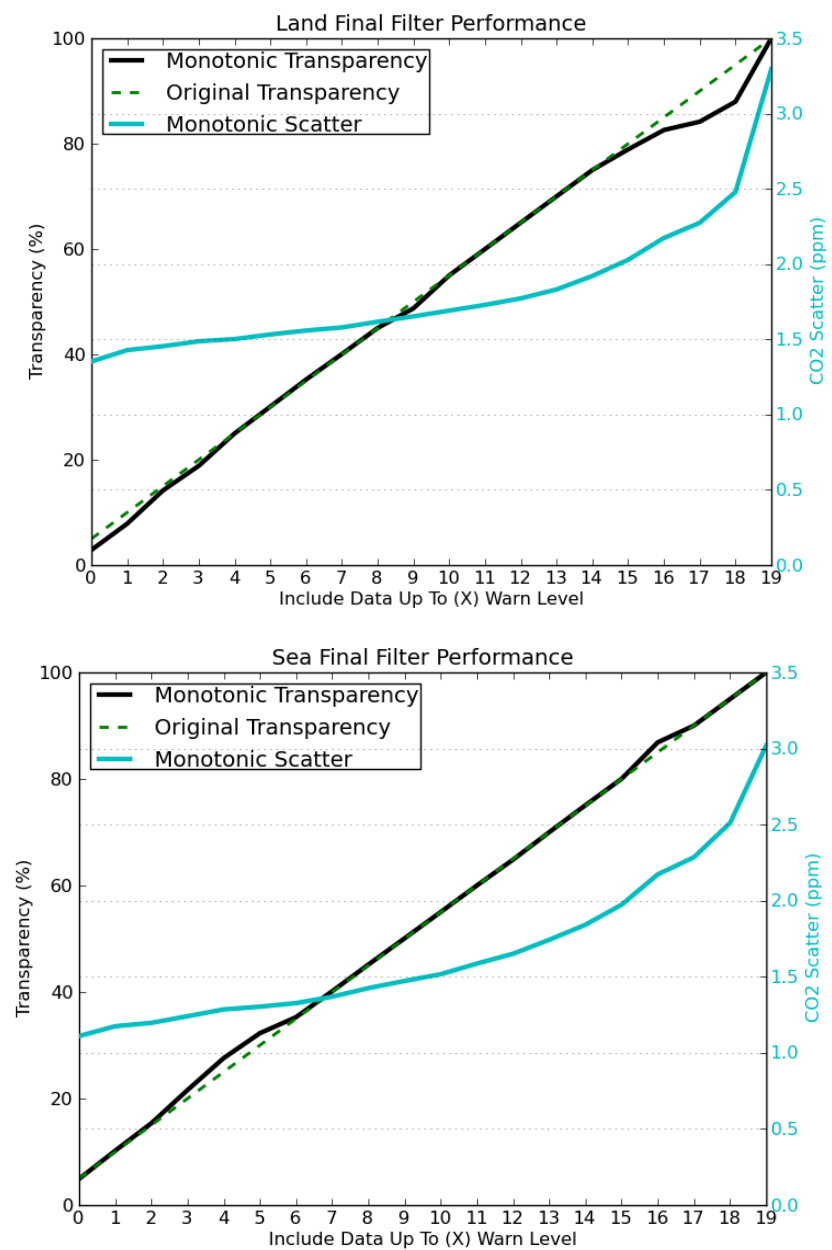

Fig. 13. The selector performance for transparency and scatter as a function of inclusive warn levels. Permitting only warn level $=0$ data (left of graph) is the most restrictive mode of the filter, while accepting everything up through warn level 19 permits all the data to the right. Note the deviation from original transparency as a function of warn level, caused by enforcing monotonicity of filter thresholds, is very small.

Examining the distribution of $\mathrm{CO} 2 \mathrm{r}$ versus $\mathrm{dPc}$ yields the decision boundary space fundamental to our future sounding selector (Fig. 9). We observe that the land has a much more dynamic $\mathrm{CO} 2 \mathrm{r}$ range, while the sea shows strong bias in $\mathrm{dPc}$ relative to land. The cutoff used to determine cloudiness over the ocean is also observable for values less than -2600 Pascals.

\subsubsection{Targeted rerun}

Permitting only $\mathrm{CO} 2 \mathrm{r}$ and $\mathrm{dPc}$ as input features, the GA is rerun with results in Fig. 10. The MMS reduction as a function of transparency remains virtually identical to the full-feature cases in Figs. 7 and 8, proving the feature selection was successful in isolating informative features. 

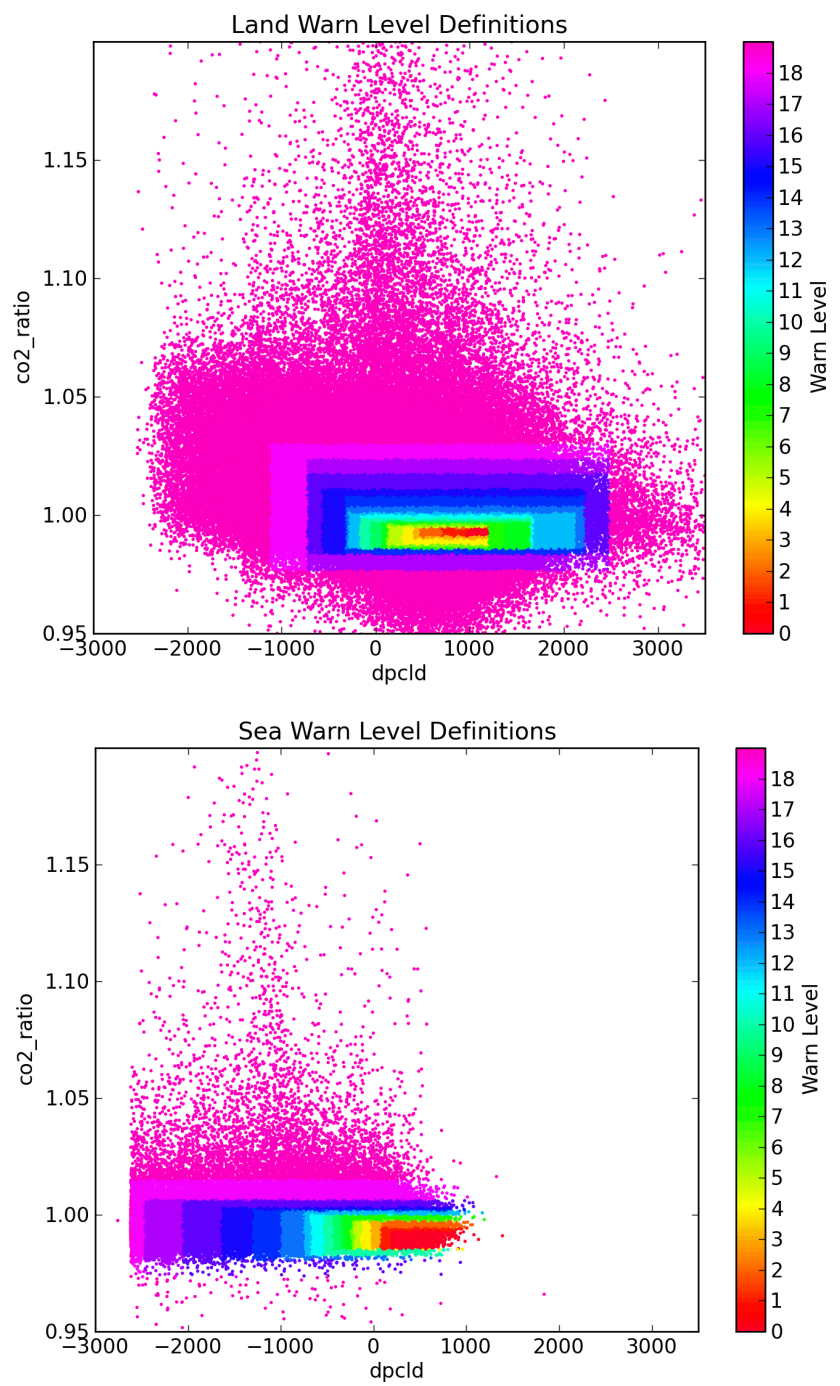

Fig. 14. The definition of warn level as a function of $\mathrm{CO} 2 \mathrm{r}$ and $\mathrm{dPc}$. The optimal values of $\mathrm{CO} 2 \mathrm{r} \sim 0.99$ and $\mathrm{dPc} \sim 800 \mathrm{hPa}$ are different from 1.0 and 0.0 due to imperfect spectroscopy.

\subsection{Warn levels}

To ensure we adequately represent the trade-off curves of Fig. 10 without having to compute a thousand filters per data point, we chose to sample the filters every $5 \%$ transparency yielding 19 uniformly spaced dominant filters corresponding to the vertical graph lines of the figure. In Figs. 11 and 12 are shown the matching high and low thresholds for the subfilters for both $\mathrm{CO} 2 \mathrm{r}$ and $\mathrm{dPc}$ as a function of transparency. The leftmost region corresponding to low transparency show considerable noise, as the low number of data being accepted results in low N statistics for the MMS. Noise in the right-most region of the graphs stems from some filter thresholds not being actively used, as other thresholds dominate this high pass rate region such as for $\mathrm{CO} 2 \mathrm{r}$ lower boundary for land or sea. Other more complex features such as near transparency $90 \%$ in the land case result from the interplay between thresholds. Left of this point, all four thresholds are cooperatively working to reduce scatter; however, to the right only the low threshold for dPc significantly contributes. There is nothing fundamentally inconsistent or problematic with such structure from the point of view of the GA, as at each step a filter was generated that satisfied the scatter reduction and transparency requirements. However, we do require some additional properties for these threshold curves for a wellbehaved sounding selector.

The uniformly sampled threshold values taken to implement the warn levels are shown as black circles in Figs. 11 and 12. Note that while following the general trend of the GA solutions, they have been made monotone as a function of transparency. This will partially violate the precise relationship of warn level to transparency percentage, but will grant a very useful property that we will name selector stability: a selector is stable if for a given transparency all the data passed at lower transparencies is included. This prevents an individual sounding from being included at low transparencies, excluded at moderate transparencies, then included again at high transparencies. Such stuttering is admissible to the GA but makes defining a unique data grouping such as is required for the warn level impossible.

The final relationship of WL versus MMS and transparency can be found in Fig. 13. The desired transparency as a function of WL, initially defined as increments of $5 \%$, is closely approximated by our monotone filter. The relationship between $\mathrm{dPc}, \mathrm{CO} 2 \mathrm{r}$, and WL for all global data (not merely the southern training data) is made clear in the decision boundary space of Fig. 14. Higher dimensional filters (complexity $>2$ ) cannot be plotted so easily but are still quite useful and not significantly harder to derive by this same procedure.

Now that we have defined our warning levels, we can discard the ensemble of fixed filters and construct our sounding selector using only desired spatial coverage and the WL labels for each sounding. It should be stated that we make an assumption at this step that the thresholds we have defined in the Southern Hemisphere will also apply to the Northern Hemisphere.

\subsection{Spatial coverage}

As an example, we test our new sounding selector using a desired transparency of $20 \%$ in Fig. 15. Shown in color are the highest (worst) warn levels per regional bin required for proper filling, where hot colors represent high quality data and cool suggest troublesome soundings with confounding factors. Land is shown only as the sea distribution is dominated by the glint geometry and is not insightful. Note that tropical regions with typically high cloudy scene likelihoods show high warn levels (Guerlet et al., 2013), while broad flat regions in the mid latitudes show ease of population with low warn level data. Shorelines and other highly mixed/complex 


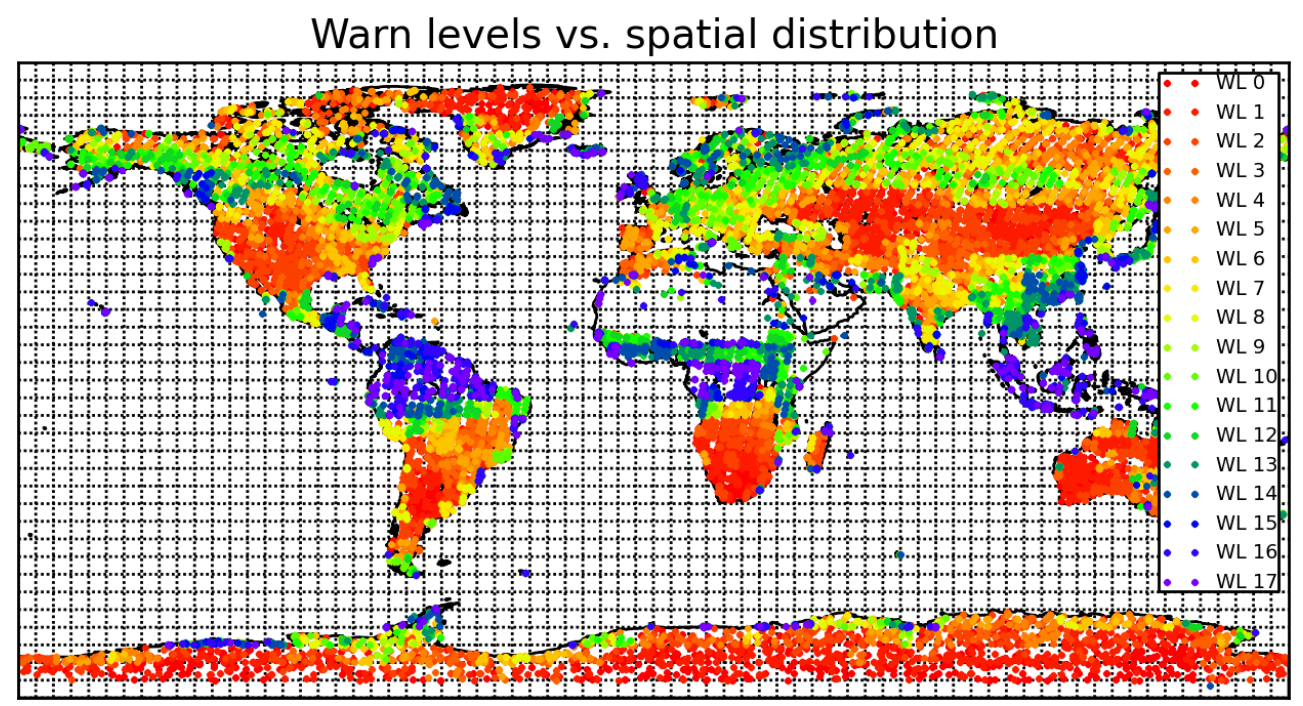

Fig. 15. Selector spatial coverage, color-coded by worst selected warn levels required for each five degree latitude region for an example $20 \%$ transparency setting using actual GOSAT data (full data set). Note that cloudy regions near the equator and highly mixed terrain such as shorelines are more problematic than large, flat regions. The unfilled regions in Africa and the Arabian Desert are due to the omission of M gain data from this analysis. Warn Level $18 \& 19$ are never permitted to be selected, as they are too confounded to be useful for later analysis.

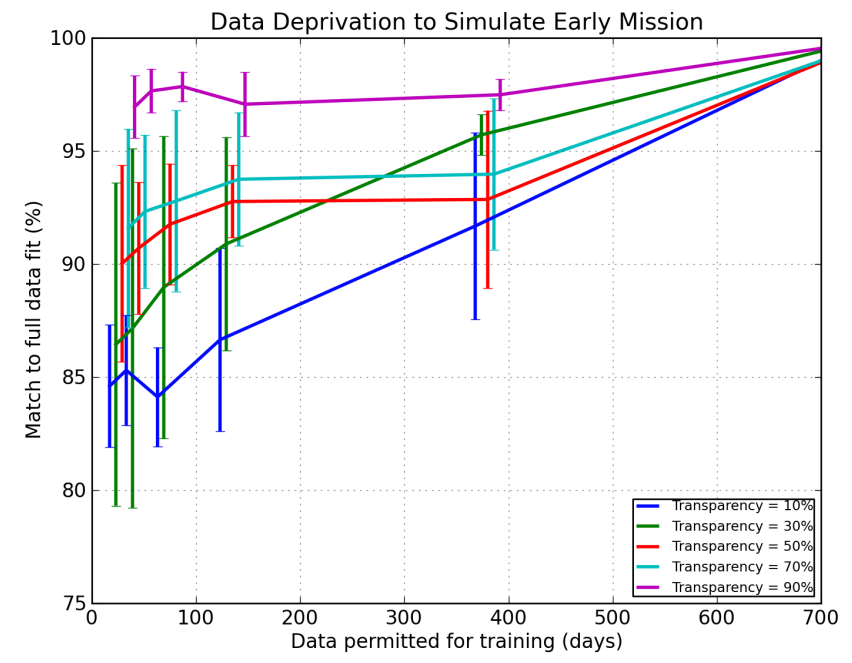

Fig. 16. Using only two weeks of randomly selected training data, at least $85 \%$ and up to $96 \%$ of the optimal sounding selector was reconstructed. Note that the $x$ axis has been shifted by a few days for each successive transparency for readability. The error bars represent the stddev of the full data match $\%$ for the set of subwindows sampled.

terrain also show an increase in warn level. The large unfilled region in Africa and the Arabian desert is due to the omission of M-gain data from this analysis, not a failure of the sounding selector. We did not permit the selector to use any warn level 18 or 19 data, as it was judged of too poor quality to be desirable in any circumstance. Such soundings typically occur in the very challenging polar regions where low solar angle, icy surfaces, and low-lying clouds conspire to prevent quality retrievals.

The confounding forces of cloud and complex terrain are well represented by the features we chose of $\mathrm{CO} 2 \mathrm{r}$ and $\mathrm{dPc}$. If we increased the complexity of the fixed filters admitted into our sounding selector construction above two, we could also use terrain roughness (filters mountainous regions) and overall spectral intensity (filters icy regions) as previously selected by the GA.

\subsection{Required data volume, early mission}

In this example, we benefitted from several years of ACOS data with which to construct an optimal sounding selector. However, when this method is applied to the upcoming OCO-2 mission, little pre-existing data will be available. Fig. 16 shows the results of constructing sounding selectors deprived of the full ACOS data set. Hundreds of subsamples of size two weeks, one month, two months, four months, or one year were randomly extracted from the data set, sounding selectors trained on each subset, and compared with the sounding selector resulting from training on the entire data set. We then compare the resulting sounding selector to the full data case in terms of percent match for included/excluded soundings. This was done repeatedly, for various selector transparencies. For low-transparency selectors, agreement with the full data set-derived filter was the most challenging at $85 \%$ using two weeks of data, while high-transparency filters were comparatively easy to reproduce even with only two weeks of data at $96 \%$ agreement. Additional training days increased the accuracy of match to 
within error. We therefore conclude that a well-functioning sounding selector can be constructed after fully processing two weeks of data at the ACOS sampling rate of five seconds. As this sampling rate is sixty times slower than OCO2 , we should need only initially process $2 \%$ of the incoming OCO-2 data for two weeks before the first-pass sounding selector can be produced. As we anticipate processing $6 \%$, there should be ample data within a few weeks for selector construction.

\section{Summary}

We have presented a procedure by which a sounding selector may be generated using the output of a genetic algorithm (GA) that seeks to reduce the mean monthly scatter (MMS) in the Southern Hemisphere. The sounding features selected as most informative by the GA are hand-reviewed to optimize interpretability and reject undesired distortion of the data, making the method semi-autonomous. Any number of informative features may be selected at this point; in the ACOS example, more than four was found to add negligible new information. Adding features (higher complexity) reduces the simplicity of the sounding selector but can encompass additional confounding forces and better fit the data. A uniform selection of fixed filters as a function of filter transparency (19 in the ACOS example) is then taken, and the thresholds of their subfilters are made monotone in transparency to preserve selector stability. This ensures that any data point permitted at a lower transparency will still be permitted at a higher transparency. The ensemble of uniformly sampled fixed filters is then used to define a warn level for each sounding equal to the number of filters that would reject it. Finally, we couple these warning levels to a spatial coverage routine that fills latitudinal bins with the lowest warn levels available. The final product is a sounding selector with a tunable transparency that maximizes the likelihood of valid $\mathrm{CO}_{2} \mathrm{re}-$ trievals from the selected soundings while guaranteeing spatial coverage. As an example point, given a $10 \%$ throughput (transparency), the Land case's mean monthly stdev (MMS) was reduced from 3.25 to $1.4 \mathrm{ppm}$, while the Sea case's MMS was reduced from 3 to $1.2 \mathrm{ppm}$. However, $10 \%$ is merely for example, and any requested percentage (rounded to the nearest $5 \%$ ) could be entered, yielding the list of best soundings to use.

This method is data-driven and agnostic to instrument. Any data set in which a goal function can be identified (i.e., the minimization of southern $\mathrm{CO}_{2}$ scatter in our case) and informative measurement features are present can be addressed in the same manner, even if the identities of the informative features are not known beforehand. Further, the execution of this method has been used to shed light onto the particular confounding forces acting upon the measurements by examining the selected most informative features and examining their spatial and temporal distribution. This same technique is now also implemented as a sounding quality estimation using post-retrieval features as well as those included here.

Acknowledgements. The research described in this paper was carried out at the Jet Propulsion Laboratory, California Institute of Technology, under a contract with the National Aeronautics and Space Administration.

Edited by: H. Worden

\section{References}

Boesch, H., Backer, D., Connor, B., Crips, D., and Miller, C.: Global Characterization of $\mathrm{CO}_{2}$ Column Retrievals from Shortwave-Infrared Satellite Observations of thet Orbiting Carbon Observatory-2 Mission, Remote Sens., 3, 270-304, 2011.

Conner, B., Boesch, H., Toon, G., Sen, B., Miller, C., and Crisp, D.: Orbiting Carbon Observatory: Inverse method and prospective error analysis, J. Geophys. Res.-Atmos., 113, D05305, doi:10.1029/2006JD008336, 2008.

Crisp, D., Atlas, R. M., Breon, F.-M., Brown, L.R., Burrows, J. P., Ciais, P., Connor, B. J., Doney, S. C., Fung, I. Y., Jacob, D. J., Miller, C. E., O’Brien, D., Pawson, S., Randerson, J. T., Rayner, P., Salawitch, R. J., Sander, S. P., Sen, B., Stephens, G. L., Tans, P. P., Toon, G. C., Wennberg, P. O., Wofsy, S. C., Yung, Y. L., Kuang, Z., Chudasama, B., Sprague, G., Weiss, B., Pollock, R., Kenyon, D., and Schroll, S.: The Orbiting Carbon Observatory (OCO) mission, Adv. Space Res., 34, 700-709, 2004.

Crisp, D., Fisher, B. M., O’Dell, C., Frankenberg, C., Basilio, R., Bösch, H., Brown, L. R., Castano, R., Connor, B., Deutscher, N. M., Eldering, A., Griffith, D., Gunson, M., Kuze, A., Mandrake, L., McDuffie, J., Messerschmidt, J., Miller, C. E., Morino, I., Natraj, V., Notholt, J., O’Brien, D. M., Oyafuso, F., Polonsky, I., Robinson, J., Salawitch, R., Sherlock, V., Smyth, M., Suto, H., Taylor, T. E., Thompson, D. R., Wennberg, P. O., Wunch, D., and Yung, Y. L.: The ACOS $\mathrm{CO}_{2}$ retrieval algorithm - Part II: Global $\mathrm{X}_{\mathrm{CO}_{2}}$ data characterization, Atmos. Meas. Tech., 5, 687707, doi:10.5194/amt-5-687-2012, 2012.

Frankenberg, C., Platt, U., and Wagner, T.: Iterative maximum a posteriori (IMAP)-DOAS for retrieval of strongly absorbing trace gases: Model studies for $\mathrm{CH}_{4}$ and $\mathrm{CO}_{2}$ retrieval from near infrared spectra of SCIAMACHY onboard ENVISAT, Atmos. Chem. Phys., 5, 9-22, doi:10.5194/acp-5-9-2005, 2005.

Guerlet, S., Butz, A., Schepers, D., Basu, S., Hasekamp, O. P., Kuze, A., Yokota, T., Blavier, J.-F., Deutscher, N. M., Griffith, D., W., T., Hase, F., Kyro, E., Mornio, I., Sherlock, V., Sussman, R., Galli, A., and Aben, I.: Impact of aerosol and thin cirrus on retrieving and validating $\mathrm{X}_{\mathrm{CO}_{2}}$ from GOSAT shortwave infrared measurements, J. Geophys. Res.-Atmos., 118, 4887-4905, doi:10.1002/jgrd.50332, 2013.

Guyon, I. and Elisseeff, A.: An Introduction to Variable and Feature Selection, J. Machine Learn. Res., 3, 1157-1182, 2003.

Jin, Y. and Sendhoff, B.: Pareto-based multi-objective machine learning: An overview and case studies, IEEE Trans. Systems, Man, and Cybernetics, Part C: Applic. Rev., 38, 397-415, 2008.

O’Brien, D. M., Pollock, R., Polonsky, I., and Rogers, M.: Identification and Correction of Residual Image in the $\mathrm{O}_{2}$ A-Band of 
the Orbiting Carbon Observatory, IEEE Trans. Geosci. Remote Sens., 49, 2426-2437, 2011.

O’Dell, C. W., Connor, B., Bösch, H., O’Brien, D., Frankenberg, C., Castano, R., Christi, M., Eldering, D., Fisher, B., Gunson, M., McDuffie, J., Miller, C. E., Natraj, V., Oyafuso, F., Polonsky, I., Smyth, M., Taylor, T., Toon, G. C., Wennberg, P. O., and Wunch, D.: The $\mathrm{ACOS} \mathrm{CO}_{2}$ retrieval algorithm - Part 1: Description and validation against synthetic observations, Atmos. Meas. Tech., 5, 99-121, doi:10.5194/amt-5-99-2012, 2012.

Periaux, J. and Galan, M.: Genetic Algorithms in Engineering and Computer Science, John Wiley \& Son Ltd, 1995.

Reuter, M., Bovensmann, H., Buchwitz, M., Burrows, J. P., Connor, B. J., Deutscher, N. M., Griffith, D. W. T., Heymann, J., Keppel-Aleks, G., Messerschmidt, J., Notholt, J., Petri, C., Robinson, J., Schneising, O., Sherlock, V., Velazco, V., Warneke, T., Wennberg, P. O., and Wunch, D.: Retrieval of atmospheric $\mathrm{CO}_{2}$ with enhanced accuracy and precision from SCIA- MACHY: Validation with FTS measurements and comparison with model results, J. Geophys. Res., 116, D04301, doi:10.1029/2010JD015047, 2011.

Taylor, T. E., O’Dell, C. W., O’Brien, D. M., Kikuchi, N., Yokota, T., Nakajima, T. Y., Ishida, H., Crisp, D., and Nakajima, T.: Comparison of Cloud-Screening Methods Applied to GOSAT NearInfrared Spectra, IEEE Trans. Geosci. Remote Sens., 50, 295309, 2012.
Wunch, D., Wennberg, P. O., Toon, G. C., Connor, B. J., Fisher, B., Osterman, G. B., Frankenberg, C., Mandrake, L., O’Dell, C., Ahonen, P., Biraud, S. C., Castano, R., Cressie, N., Crisp, D., Deutscher, N. M., Eldering, A., Fisher, M. L., Griffith, D. W. T., Gunson, M., Heikkinen, P., Keppel-Aleks, G., Kyrö, E., Lindenmaier, R., Macatangay, R., Mendonca, J., Messerschmidt, J., Miller, C. E., Morino, I., Notholt, J., Oyafuso, F. A., Rettinger, M., Robinson, J., Roehl, C. M., Salawitch, R. J., Sherlock, V., Strong, K., Sussmann, R., Tanaka, T., Thompson, D. R., Uchino, O., Warneke, T., and Wofsy, S. C.: A method for evaluating bias in global measurements of $\mathrm{CO}_{2}$ total columns from space, Atmos. Chem. Phys., 11, 12317-12337, doi:10.5194/acp11-12317-2011, 2011.

Yokota, T., Oguma, H., Morino, I., and Inoue, G.: A nadir looking SWIR FTS to monitor $\mathrm{CO}_{2}$ column density for Japanese GOSAT project, Proc. Twenty-fourth Int. Sympo. on Space Technol. and Sci., JSASS and Organizing Comm. of the 24th ISTS, 887-889, 2004. 